\title{
Nucleon number dependence of longitudinal radii in ion-ion collisions as a signature of the onset of collective expansion
}

\author{
Ján Pišút ${ }^{a b b)}$, Neva Pišútováb) and Petr Závada ${ }^{c)}$ \\ a)Laboratoire de Physique Corpusculaire,Université Blaise Pascal, \\ Clermont-Ferrand, F-63177 Aubière, Cedex, France \\ b) Department of Physics, Comenius University, SK-842 15 \\ Bratislava,Slovakia \\ c)Institute of Physics, Czech Acad.Sci. Na Slovance 2, CZ-18040, Praha-8 \\ Czech Republic
}

\begin{abstract}
In an attempt to disentangle the effects of nuclear geometry from those of expansion we study the dependence of the longitudinal radius $\left(R_{L}\right)$ on nuclear numbers of colliding ions within a simple model of multiple nucleon- nucleon collisions. The model has built in the nuclear geometry but there is no dynamical expansion.In principle, what is significantly beyond models of this type can be considered as caused by collective expansion. Taking into account the present errors of data, oversimplified model and the absence of accurate information on $R_{L}$ for pp interactions it is difficult to make strong statements about collisions of induced by light ions. On the other hand $\mathrm{Pb}-\mathrm{Pb}$ data give $R_{L}$ significantly higher than a geometrical picture could accomodate, presenting an evidence for the onset of collective expansion, or of onset of a new dynamical regime between $\mathrm{O}-\mathrm{Au}$ and $\mathrm{Pb}-\mathrm{Pb}$ collisions. The situation with $\mathrm{S}-\mathrm{Au}$ and $\mathrm{S}-\mathrm{Pb}$ collisions is less clear because of a rather large difference of data on $R_{L}$ in these two cases.
\end{abstract}




\section{Introduction}

Heavy ion collisions provide the only way to laboratory studies of dense hadronic matter and hopefully also of theoretically predicted Quark- Gluon Plasma $(Q G P)$. Hanbury- Brown and Twiss $(H B T)$ interferometry $[1,2]$ brings information about dimensions of the homogeneity region $[3,4,5]$ of particle production.

Space-time evolution of proton-nucleus $(p A)$ and of ion-ion $(A B)$ collisions depends on details of dynamics and on values of important parameters,like the formation time of secondary hadrons, which are both only partially understood.It is therefore impossible to predict accurately when the collective expansion of hadronic matter or of $Q G P$ sets on.In an ideal situation dynamics of heavy-ion collisions would be known up to a few parameters which could be determined by HBT interferometry. Unfortunately the dynamics is unknown and because of that we shall start with simplest assumptions possible.

We suppose that in the CERN SPS energy range collisions of protons and of light ions with nuclear targets consist of multiple nucleon-nucleon collisions, with dynamics close to that described in Ref.[6] . For earlier work in this direction, see Ref.[7] .In each of collisions, except for the last one, nucleon loses some fraction of its energy.In the last one it fragments roughly like in a $p p$ collision. In those kinematical regions where the production of secondary particles is dominated by last collisions of individual nucleons, the model of Ref. [6] is close to predictions of the "wounded" nucleon [8] or "participating" nucleon [9] models. The model of Ref.[6] admits a parton model interpretation, according to which fast partons, including valence quarks, do not participate in individual nucleon-nucleon collisions, what makes the picture consistent [10] with the experimental information on the A,B dependence of the Drell-Yan dilepton production. This model is not exactly that of multiple nucleon-nucleon scattering in the whole rapidity domain.For secondary hadrons in the central rapidity region the picture might be close to that of independent nucleon- nucleon collisions,since slow hadrons in the nucleonnucleon cms might be formed in between of successive collisions.

The purpose of the present note is to discuss a formula for the dependence of $R_{L}$ on nucleon numbers $A$ and $B$ of colliding ions,based on the picture of multiple nucleon-nucleon collisions. The formula might roughly correspond also to other models,provided that the collective expansion is rather small. 
We shall then compare the formula with available data [11-14] of NA-44, NA-35 and WA-93 Collaborations obtained at the CERN-SPS and try to see whether the data can be understood only by nuclear geometry and fragmentation of nucleons or whether some dynamical expansion is required.We shall use two approaches.In the former one we assume that expanding hadronic matter is not present in proton and light ion collisions with nuclei in the CERN SPS energy region. The parameters in the model are then determined from this condition. Having parameters fixed in this way we compare the formula with data on $R_{L}$ obtained in heavy ion collisions in particular in $\mathrm{Pb}+\mathrm{Pb}$ and $S+\mathrm{Pb}$ ones.If values of $R_{L}$ in these collisions are not larger than what follows from such an extrapolation of data from lower A's and B's then also $\mathrm{S}+\mathrm{Pb}$ and $\mathrm{Pb}+\mathrm{Pb}$ collisions would be most likely dominated just by nuclear geometry.If,on the other hand,longitudinal radii as measured in $\mathrm{S}+\mathrm{Pb}$ and $\mathrm{Pb}+\mathrm{Pb}$ are larger than such extrapolations, then a new dynamical mechanism,presumably caused by the collective expansion of matter, must be responsible for the increase of $R_{L}$.

In the latter approach we take the model literally and calculate the parameters.Deviations from the model are supposed to give evidence for the presence of expansion.

In both approaches we are trying to find a threshold at which $R_{L}$ "decouples" from simple geometrical dependence.Any point of decoupling or a point when one type of behaviour of $R_{L}$ changes to another one is most likely related to the onset of a new dynamics of the collision.A threshold for "decoupling" from a simple geometrical dependence signals most probably a transition to a collective expansion of matter.

Note that such thresholds should be correlated also with changes of patterns of other signatures,like enhancement of dilepton and photon production, increased $J / \psi$ suppression,appearance of azimutal asymmetries in noncentral collisions,etc. 


\section{Dependence of $R_{L}$ on $\mathrm{A}$ and $\mathrm{B}$ in a model of multiple nucleon- nucleon collisions}

The correlation function for two identical pions with momenta $\vec{k}_{1}$ and $\vec{k}_{2}$ is written in the standard way

$$
C\left(\vec{k}_{1}, \vec{k}_{2}\right)=1+\lambda\left|\int e^{i\left(\vec{k}_{1}-\vec{k}_{2}\right) \cdot \vec{r}} \rho(\vec{r} ; \vec{K}) d^{3} \vec{r}\right|^{2}
$$

where $\vec{K}=\left(\vec{k}_{1}+\vec{k}_{2}\right) / 2$ and $\rho(\vec{r} ; \vec{K})$ is the density of sources of the corresponding homogeneity region $[3,4,5]$. To simplify the discussion we have assumed that both pions have the same energy.In that case $\rho(\vec{r} ; \vec{K})$ in Eq.(1) is integral over time of the space-time density distribution $\rho(\vec{r}, t ; \vec{K})$. The density of sources $\rho(\vec{r} ; \vec{K})$ is asummed to be given, in some approximation, as

$$
\rho(\vec{r} ; \vec{K})=\frac{1}{R_{T}{ }^{2} R_{L}(2 \pi)^{3 / 2}} \exp \left(-\frac{z^{2}}{2 R_{L}{ }^{2}}-\frac{x^{2}+y^{2}}{2 R_{T}{ }^{2}}\right)
$$

The Fourier transform corresponding to $\vec{k}_{1}-\vec{k}_{2} \equiv \vec{q}$ parallel to the beam $(\equiv z)$ axis becomes

$$
\tilde{\rho}(\vec{q})=\exp \left(-\frac{R_{L}^{2} q^{2}}{2}\right)
$$

and the correlation function in Eq.(1) is

$$
C(q)=1+\lambda \exp \left(-R_{L}^{2} q^{2}\right)
$$

The longitudinal radius $R_{L}$ is related to the mean squared value of $z$ by

$$
R_{L}^{2}=\left\langle z^{2}\right\rangle=\int z^{2} \rho(\vec{r} ; \vec{K}) d^{3} \vec{r}
$$

We shall further assume that in individual nucleon-nucleon collisions pions with small $z$-component of momentum (in the nucleon-nucleon c.m.s.) are produced in a way similar to the one in collisions of free nucleons. The process goes pressumably via formation and decay of resonances in both cases. We thus assume that the density of sources for identical pions in a given nucleonnucleon collision which took place in the point with coordinate $\bar{z}$ is given as

$$
\rho_{n n}(z-\bar{z} ; \vec{K})=\frac{1}{R_{L}(p p)(2 \pi)^{1 / 2}} \exp -\left(-\frac{(z-\bar{z})^{2}}{2 R_{L}^{2}(p p)}\right)
$$


Here $R_{L}(p p)$ is the longitudinal radius as obtained by HBT interferometry in $p p$ collisions. The experimental information obtained by the AFS Collaboration at the CERN ISR [15] indicates that $R_{L}(p p)$ is a bit larger than $1 \mathrm{fm}$. This result has been corroborated by further evidence obtained in less direct measurements [16] in studies of directional dependence of HBT interferometry,based on the method proposed in Ref.[17]. The density of nucleonnucleon collisions is parametrized as

$$
\rho_{l}(\bar{z})=\frac{1}{l \sqrt{2} \pi} \exp \left(-\frac{\bar{z}^{2}}{2 l^{2}}\right)
$$

Where $l$ is given by Eq.(6). The total density of sources is then given as

$$
\rho(z ; \vec{K})=\int \rho_{n n}(z-\bar{z} ; \vec{K}) \rho_{l}(z-\bar{z}) d \bar{z}
$$

where we have suppressed variables $x, y$.The Fourier transform of a convolution is the product of Fourier transforms and this leads via Eqs. $(1,7,8,9)$ to

$$
C(q ; \vec{K})=1+\lambda \exp \left(-\left(l^{2}(A, B)+R_{L}^{2}(p p)\right) q^{2}\right)
$$

and finally to

$$
R_{L}^{2}(A, B)=R_{L}^{2}(p, p)+l^{2}(A, B)
$$

Where $l^{2}(A, B)$ is given in Eq.(7) and can be calculated as

$$
l^{2}(A, B)=<\bar{z}^{2}>
$$

The interpretation of Eq.(10) is simple. The experimentally observable longitudinal radius in $A B$ collisions consists of two parts. The former, $R_{L}(p p)$ is due to resonance decays or to equivalent dynamical reasons (production of clusters,etc.) and the latter is due to the geometry of the $A B$ collision.

\section{Two approaches to the comparison of the formula with data}

We shall now compare the formula in Eq.(10) with data within the two approaches discussed in the Introduction. 
The former approach is more qualitative.We assume that in $A+B$ collision individual nucleon-nucleon interactions occur in a region with longitudinal dimension

$$
l \approx \operatorname{cr}_{0} \frac{1}{\gamma}\left((A-1)^{1 / 3}+(B-1)^{1 / 3}\right)
$$

Here $r_{0} \approx 1.2 \mathrm{fm}, \gamma$ is the Lorentz contraction factor, $c$ is a constant of the order 1 and replacements $A \rightarrow(A-1), B \rightarrow(B-1)$ are unimportant for the data we shall discuss,but they facilitate the transition to $p p$ collisions. The constant $c$ may include some details of dynamics as well as the fact that the approximation $R_{A} \approx 1.2 r_{0} A^{1 / 3}$ underestimates [18] radii of light ions. Conventions used correspond to those of NA-44 and we have recalculated the NA-35 data accordingly.

We shall consider the interference of identical pions with $K_{z} \approx 0$ in the c.m.s. of nucleon-nucleon collisions. In this frame incoming nuclei appear contracted by the Lorentz factor $\gamma=s^{1 / 2} / 2 M$ where $s$ is the square of the nucleon-nucleon c.m.s. energy and $M$ is the nucleon mass.For $E_{L}=$ $200 \mathrm{AGeV}, \gamma \approx 10$ and for $E_{L}=160 \mathrm{AGeV}$ we have $\gamma \approx 9$.

From Eqs.(10) and (12) we find

$$
R_{L}{ }^{2}(A, B)=R_{L}{ }^{2}(p p)+\left(\left(c r_{0} / \gamma\right)\left((A-1)^{1 / 3}+(B-1)^{1 / 3}\right)\right)^{2}
$$

For $A=B=1$ we obtain $R_{L}(1,1)=R_{L}(p, p)$ as we should.

The comparison of data on $R_{L}(A, B)$ as obtained at the CERN SPS with Eq.(13) is presented in Fig.1.The data corresponding to $A B$ collisions were taken from the following sources: $S+C$ from Ref.[11], $S+S$ from Ref.[11]; $p+P b$ from Ref.[13]; $S+C u$ from Ref.[11]; $S+A g$ from Ref.[11]; $O+A u$ from Ref.[11]; $S+A u$ from Ref.[11]; $S+P b$ from Ref.[13] and $P b+P b$ from Refs. $[13,14]$.

The straight line in Fig.1 corresponds to the following values of parameters entering Eq.(11)

$$
R_{L}(p p) \approx 1.92 \mathrm{fm} ; \quad \frac{c r_{0}}{\gamma} \approx 0.36 \mathrm{fm}
$$

Since we have read the data of Ref.[13] from graphs and since the statistics is continuously increasing we do not give errors of the fit of data by 
Eq.(11),pointing out only that the determination of $R_{L}(p p)$ by the data is less accurate than that of $c r_{0} / \gamma$.

The qualitative, and in our opinion the most important point, is easily visible in Fig.1. The data from $S+C$ up to $O+A u$ are compatible with the straight line whereas $R_{L}(P b P b)$ is certainly much larger than what could be ascribed to the linear dependence obtained from proton- and light ion collisions with nuclear targets. We interpret this as an evidence for the presence of a threshold occuring between $\mathrm{O}+\mathrm{Au}$ and $\mathrm{Pb}+\mathrm{Pb}$ collisions at the CERN SPS energies.Fig. 1 contains also an indication of a possible discrepancy between data on $R_{L}$ on $S+A u$ as given in Ref.[11] and on $S+P b$ presented in Ref.[13]. Difference between nucleon numbers of $A u(197)$ and $P b(207)$ is hardly responsible for a large difference between the corresponding values of $R_{L}$. If the true value of $R_{L}(S P b)$ is closer to Ref.[13] the threshold is probably around $S+P b$ collisions.

The value of $R_{L}(p p) \approx 1.92 \mathrm{fm}$ seems to be larger than expected,but since this is not very accurately determined by the "fit" we shall not try to analyze possible reasons for that.

Taking $\gamma \approx 10$ and $r_{0} \approx 1.2 \mathrm{fm}$ we find $c \approx 3$ what is also somewhat large. The value of $c$ might be also influenced by the underestimate of radii of light ions by the expression $R_{A}=r_{0} A^{1 / 3}$,see Ref.[18].

Note that in the region where data in Fig.1 are presented the dependence as given by Eq.(11) is not much different from a linear relationship between $R_{L}(A B)$ and $(A-1)^{1 / 3}+(B-1)^{1 / 3}$.

Arguments given above are admittedly rather crude.In spite of simplifications used we shall make an attempt to interpret the results. The experimental value $[13,14]$ of the square of longitudinal radius for $P b+P b$ collisions is about $40 \mathrm{fm}^{2}$, whereas the extrapolation from proton- and light ion nuclear collisions gives about $20 \mathrm{fm}^{2}$. The expansion should be responsible for the difference.Putting $R_{L, \text { exper }}^{2} \approx R_{L, \text { geom }}^{2}+R_{\text {expan }}^{2}$ we get $R_{\text {expan }} \approx 6 \mathrm{fm}$. Since $\left\langle z^{2}\right\rangle=$ $R_{L}{ }^{2}$ we have $\left\langle z^{2}\right\rangle_{\text {expan }}^{1 / 2} \approx 5.6 \mathrm{fm}$. Assuming that for pions with $K_{z} \approx 0$ the homogeneity region corresponds roughly to rapidity region $-1 / 2<y<1 / 2$ than the time of expansion is roughly $t_{\text {expan }} \approx\left\langle z^{2}\right\rangle_{\text {expan }}^{1 / 2} / v(y=1 / 2)$ where $v(y=1 / 2)=\tanh (1 / 2)$ is the velocity corresponding to $y=1 / 2$.In this way we get the estimate $t_{\text {expan }} \approx 11.2 \mathrm{fm} / \mathrm{c}$.

The latter approach is more rigid. Values of $l^{2}(A+B)$ entering Eqs.(10) and (11) are obtained from nuclear geometry without any free constants. We 
have calculated values of $l^{2}(A+B)$ for different colliding nuclei by using the model of Ref.[19].In this model distribution of nucleons in nuclei is given by a standard Woods- Saxon density. The resulting values of $l^{2}(A+B)$ for central collisions in the c.m.s of nucleon- nucleon interactions and taking into account Lorentz contraction are as follows (in $\mathrm{fm}^{2}$ )

$$
\begin{array}{ccc}
l^{2}(S+C)=0.014 & l^{2}(S+S)=0.018 & l^{2}(S+C u)=0.022 \\
l^{2}(S+A g)=0.029 & l^{2}(O+A u)=0.037 & l^{2}(S+A u)=0.041 \\
l^{2}(p+P b)=0.038 & l^{2}(S+P b)=0.042 & l^{2}(P b+P b)=0.055
\end{array}
$$

In these calculations we have not taken into account deceleration of nucleons by collisions.

Very similar values of $l^{2}(A+B)$ are obtained in a simple model in which nucleus with radius $\mathrm{R}$ is replaced by a cylinder of length $2 \mathrm{~L}$ with $L=$

$\sqrt{3 / 5} R$. This relation is given by the requirement that $\left\langle z^{2}\right\rangle$ is the same in both cases.

Values in Eq.(15) are rather small with respect to $R_{L}^{2}(p p)$ and the corresponding curve in Fig. 1 is practically flat. To draw the curve we need the value of $R_{L}^{2}(p p)$. The interpretation of data is thus made difficult by the absence of experimental information on $R_{L}^{2}(p p)$.But even admitting a very large, and probably unrealistic, value of $R_{L}^{2}(p p)$, say up to about $4 \mathrm{fm}^{2}$ the data would indicate a presence of expansion even in collisions like $\mathrm{p}+\mathrm{Pb}$.Assuming a particular value of $R_{L}^{2}(p p)$ we can roughly estimate the expansion times as above. For $R_{L}(p p)=1 \mathrm{fm}$ and $R_{L}(P b+P b) \approx 6 \mathrm{fm}$ we obtain $t_{\text {expan }} \approx 12.6 \mathrm{fm} / \mathrm{c}$ for $\mathrm{Pb}-\mathrm{Pb}$ interactions and with $R_{L}(S+\mathrm{Pb}) \approx 4 \mathrm{fm}$ we have $t_{\text {expan }} \approx 8.4 \mathrm{fm} / \mathrm{c}$ for $\mathrm{S}-\mathrm{Pb}$ collisions.

\section{Comments and conclusions}

Data on $R_{L}$ obtained in ion-ion collisions at the CERN-SPS [11,12,13] give a valuable information on the space- time evolution of these collisions.We have tried above to analyze these data by using a very simple picture of ionion collision as a sum of nucleon- nucleon interactions. The picture leads to a simple formula Eq.(10).We have used this formula in two approaches.In the former we have introduced $R_{L}(p p)$ and the constant $c$ in Eq.(12) as free parameters.In this way data with incident light ions can be interpreted as 
due to nuclear geometry, but $R_{L}$ for $\mathrm{Pb}-\mathrm{Pb}$ collisions is definitely higher than a geometrical model can accomodate what gives evidence for the presence of collective expansion of matter in $\mathrm{Pb}-\mathrm{Pb}$ collisions. The case of $\mathrm{S}-\mathrm{Pb}$ and $\mathrm{S}-\mathrm{Au}$ collisions is difficult to interpret since there is an unexpectedly large difference between the two data.It turns out also that an accurate information on $R_{L}(p p)$ is of primary importance.

In the latter approach we have calculated the contribution of nuclear geometry to $R_{L}(p p)$ in a more rigid way,leaving $R_{L}(p p)$ as the only unknown parameter. The resulting $R_{L}(A B)$ as given by geometry is almost independent of $A, B$ and practically equal to $R_{L}(p p)$. The value of $R_{L}(p p)$ is thus a key to interpreting data on $R_{L}$ for light- ion induced interactions. $\mathrm{Pb}-\mathrm{Pb}$ data are again much higher than geometry can explain.Our estimates of the expansion time in $\mathrm{Pb}-\mathrm{Pb}$ collision give $t_{\text {expan }} \approx 9 \mathrm{fm} / \mathrm{c}$.

Study of the dependence of $R_{L}$ on A,B and on incident energy can, in our opinion, reveal the changing dynamics of ion- ion collisions. This concerns in particular presence of "thresholds" corresponding to changes of the dynamical regime.

The absence of accurate information on $R_{L}(p p)$ and rather large errors of data make it difficult to see whether data indicate presence of one or two "thresholds". The first one might be rather low - somewhere between pp and S-C collisions, but this question depends crucially on the value of $R_{L}(p p)$, and the second one between $\mathrm{O}-\mathrm{Au}$ and $\mathrm{Pb}-\mathrm{Pb}$. The existence of the latter seems very probable.

AcknowledgementsThe authors are indebted to C.Fabjan,D.Ferenc, R.Lietava,G.Roche and B.Tomášik for valuable discussions, to Yiota Foka for having organized a very stimulating HBT-Forum at CERN in March 1996 and to Daniel Ferenc for calling our attention to to an erroneous factor of two in some of equations. One of the authors (J.P.) is indebted to Guy Roche and Bernard Michel for hospitality at the Laboratoire de Physique Corpusculaire,Université Blaise Pascal,Clermont-Ferrand.

Figure Caption The dependence of $y=R_{L}{ }^{2}(A, B)$ as given in Refs.[11- 
14] on $x=\left((A-1)^{1 / 3}+(B-1)^{1 / 3}\right)^{2}$. Straight line fit corresponds to $y=a+b x$ with $a=3 \mathrm{fm}^{2}$ and $b=0.13 \mathrm{fm}^{2}$.In making the fit we have excluded data on $S+P b$ and $P b+P b$ collisions. Note the $P b+P b$ point in the right upper corner.For discussion of the second approach to data the reader is invited to draw a line parallel with the $\mathrm{x}$-axis and corresponding to his preferred value of $R_{L}(p p)$.

\section{References}

[1] R.Hanbury-Brown and R.Q.Twiss,Phil.Mag.45(1954)663; Nature 178 (1956) 1046.

[2] G.Goldhaber,S.Goldhaber,W.Lee and A.Pais, Phys. Rev. 120 (1960) 300.

[3] A.N.Makhlin and Yu.M.Sinyukov, Zeit. f. Phys. C39 (1988) 69;Yu.M.Sinyukov, Nuc. Phys. A498 (1989) 151c; Yu. M. Sinyukov, Nucl.Phys. A566 (1994) 589c.

[4] K.Kolehmainen and M.Gyulassy, Phys. Lett. B180 (1986) 203; B.Andersson and W.Hofmann,Phys.Lett. B169 (1986) 364; S. Padula and M.Gyulassy, Nucl.Phys. A498 (1988) 555c.

[5] T.Csörgö, Phys. Lett. B347 (1995) 354; S. Chapman, J.R.Nix, and U.Heinz, Phys. Rev. C52 (1995) 2694.

[6] S.Daté, M.Gyulassy, and H.Sumiyoshi, Phys.Rev. D32 (1985) 617.

[7] R.C.Hwa,Phys.Rev.Lett. 52 (1984) 493, J.Hüfner, and A.Klar, Phys.Lett. B145 (1984) 167; K.Kinoshita et al. Progr. Theor. Phys. 63 (1980) 928; R.C.Hwa, and M.S.Zahir, Phys. Rev. D31 (1985) 499.

[8] A.Bialas,A.Bleszynski, and W.Czyz, Nucl. Phys. B111 (1976) 461; A.Bialas, and W.Czyz, Nucl.Phys. B194 (1982) 21; A.Bialas, W.Czyz, and L.Lesniak, Phys. Rev. D25 (1982) 2328.

[9] H.Brody,S.Frankel,W.Frati, and I.Otterlund, Phys. Rev. D28 (1983) 2334; L.P. Remsberg, and M.J.Tannenbaum, Zeit. f. Phys. C38 (1988) 35; J.Ftáčnik,K.Kajantie, N.Pišútová, and J.Pišút, Phys. Lett. B188 
(1987) 279; A. Bamberger et al., NA-35 Collab., Phys. Lett. B184 (1987) 271.

[10] T.Jaroszewicz, and M.Jezabek, Zeit. f. Phys. C4 (1980) 277.

[11] T.Alber et al.,NA-35 Collab., Zeit. f. Phys. C66 (1995) 77.

[12] B.Jacak, NA-44 Collab., Nucl.Phys. A590 (1995) 215c.

[13] J.Dodd, NA-44 Collab., Talk at the 25th Int. Symposium on Multiparticle Dynamics 1995,Stará Lesná,12.-16.Sept.1995, Proc. will be published by World Scientific, Singapore, 1996, Ed. L. Šándor et al.

[14] S.Slegt et al.,WA-93 Collab., Nucl.Phys. A590 (1995) 469c.

[15] T.Akesson et al. AFS Collab., Phys.Lett. B187 (1987) 420.

[16] N.M.Agababyan et al.EHS/NA22 Collab., Zeit. f. Phys. C66 (1995) 409;

R.A.Kvatadze, R.M.Moller and B. Lörstad, Zeit. f. Phys. C38 (1988) 551.

[17] M.I.Podgoretsky, and A.P. Cheplakov, Sov. J. Nucl. Phys. 44 (1986) 835.

[18] R.A.Salmeron,Nucl.Phys. B389 (1991) 301.

[19] P.Závada,Phys.Rev. C40 (1989) 285, P.Závada, Phys. Rev. C42 (1990) 1104. 


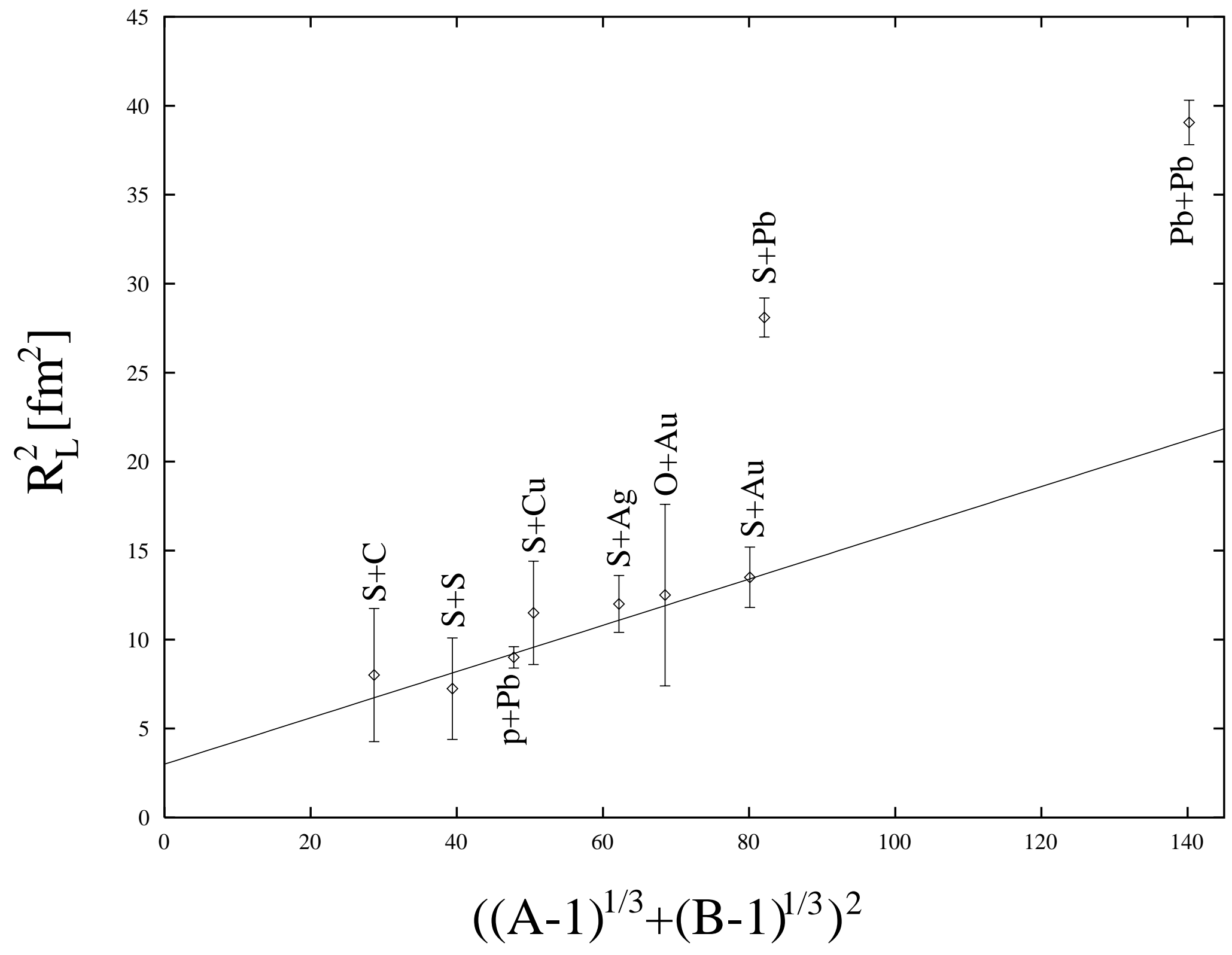

Fig.1 